\title{
LES JUNIPERETA MACROCARPAE SUR SABLE
}

\author{
Jean Marie GEHU, Manuel COSTA \& Eduardo BIONDI
}

\begin{abstract}
RESUME: Les auteurs étudient les Junipereta macrocarpae sur sable des dunes méditerranéennes. L'application des méthodes phytosociologiques actuelles permet de mettre en évidence sur les côtes méditerranéennes et les littoraux voisins quatre associations territoriales géosynvicariantes à Juniperus oxycedrus L. ssp. macrocarpa (Sibth. et Sm.) Ball possédant chacune une aire géographique particulière.
\end{abstract}

Mots clés: Juniperus oxycedrus L. ssp. macrocarpa (Sibth. et Sm) Ball, dunes méditerranéennes, géosynvicariançe.

RESUMEN: Los autores estudian laJunipereta macrocarpae sobre arena de dunas mediterráneas. La aplicación de métodos fitosociológicos actuales permiten distinguir sobre las costas mediterráneas y los litorales vecinos cuatro asociaciones territoriales geosinvicariantes con Juniperus oxycedrus L. subsp. macrocarpa (Sibth. et Sm.) Ball teniendo cada una un entorno geográfico particular.

Palabras clave: Juniperus oxycedrus L. subsp. macrocarpa (Sibth. et Sm) Ball, dunas mediterráneas, geosinvicariancia.

\section{N T R O D U C T I O N}

Juniperus oxycedrus L. ssp. macrocarpa (Sibth. et Sm.) Ball, bien que non exclusivement lié aux dunes méditerranéennes -il existe aussi sur falaises (Corse) ou dans l'intérieur des terres (Grèce, Yougoslavie)- apparaît cependant de préférence sur les sables côtiers. Il y constitue généralement le tout premier terme de boisement naturel de l'arrière-dune, résistant aux vents marins chargés de sel et de sable.

L'Atlas Florae Europaeae, donne pour l'Europe une carte de distribution de ce conifère méditerranéen qui n'inclut malheureusement pas les données nord-africaines et turques (carte $\left.\mathrm{n}^{\mathrm{O}} 1\right)$.

Dans son habitat dunaire Juniperus oxycedrus L. ssp. macrocarpa (Sibth. et Sm) Ball est associé à diverses espèces des Quercetea ilicis Br.-B1. 1947 et plus précisément des Pistacio-Rhamnetalia alaterni Riv.-Mart. 1975 et du Juniperion lyciae Riv.-Mart. 1975. Cependant de l'Ouest à l'Est méditerranéen, le contingent d'espèces associées varie, permettant de penser qu'il y existe un phénomène de géosynvicariance entre les communautés de Juniperus oxycedrus L. ssp. macrocarpa (Sibth. et $\mathrm{Sm})$ Ball selon leur position géographique. 
Le but de ce travail est de vérifier cette hypothèse tout en confrontant les données de la littérature et celles récoltées par les auteurs.

\section{E T H O D E}

Le matériel phytosociologique disponible sous forme de relevés issus de la littérature (cf. Bibliographie) ou de nos archives a été confronté d'abord en un tableau détaillé qui a été ultérieurement condensé en un tableau synthétique. Seul ce dernier est publié ici.

La synthèse du matériel phytosociologique présente toujours certaines difficultés, voire certains risques, qui proviennent essentiellement de l'hétérogénéité plus ou moins grande du milieu à analyser et des conceptions et modalités de travail des différents auteurs dont le matériel est utilisé.

Le milieu dunaire, par la dynamique mésologique qui est la sienne et les perturbations environnementales récentes qu'il connaît, est de ce point de vue particulièrement délicat à étudier.

C'est pourquoi avant d'exposer les résultats obtenus, il paraît nécessaire d'attirer l'attention du lecteur sur quelques précautions indispensables que nous nous sommes attachés à respecter.

La première est évidemment d'avoir personnellement une large expérience de terrain des communautés étudiées. Ce que nous confère en l'occurence un programme d'études en commun sur la végétation littorale périméditerranéenne entrepris depuis 10 ans. On a de la sorte la possibilité de mieux comprendre ou de mieux interpréter les données des auteurs.

Celles-ci, avant d'être utilisées, et c'est la seconde précaution, doivent être examinées à la lumière de l'expérience acquise. Relevé par relevé, il est généralement possible de déceler les relevés fragmentaires ou complexes à rejeter ou à n'utiliser qu'avec circonspection.

Nous voudrions à ce sujet introduire ici la notion de "nombre moyen corrigé d'espèces".

On sait que le nombre moyen d'espèces d'un tableau phytosociologique est obtenu par la somme des espèces des relevés de ce tableau divisée par le nombre de relevés du même tableau soit:

Nombre moyen d'espèces d'un tableau (N.m.E.t.) $=\sum$ E.t./ N.R.t.

La notion de "nombre moyen corrigé d'espèces" permet dans la confection d'un tableau synthétique à partir de données issues d'auteurs divers de corriger l'hétérogénéité induite par la prise de relevés plus ou moins "larges" sur le terrain, incluant ou non les espèces dites de "contact", c'est-à-dire appartenant à des communautés voisines ou mosaïquées.

Le nombre moyen corrigé d'espèces est obtenu par l'équation:

Nombre moyen corrigé d'espèces (N.m.Cr.E.) = Nombre moyen brut d'espèces (N.m.b.E) - Nombre moyen d'espèces de contact (N.m.E.Ct.)

$$
\text { N.m.Cr.E. = N.m.b.E - N.m.E.Ct. }
$$


Ainsi, dans le cas des Junipereta macrocarpae étudiés, le nombre moyen corrigé confirme la parenté des deux races de l'Asparago-Juniperetum macrocarpae de Bolòs 1964, alors que le nombre moyen brut donnait une information inverse par suite des nombreuses espèces de contact présentes dans les relevés de la race à Prasium majus $\mathrm{L}$. Ce qui aurait pu conduire à la définition erronée d'une association différente.

Enfin, troisième condition, les données statistiques obtenues par comparaison des relevés, c'est-à-dire les combinaisons floristiques particulières doivent chacune correspondre à une aire géographique précise et différente.

\section{R E S UL T A T S}

Le tableau de synthèse, obtenu par la confrontation de 114 relevés dont les sources sont indiquées ci-après met en évidence l'existence d'une réelle variation floristique des communautés à Juniperus oxycedrus L. ssp. macrocarpa (Sbth. et Sm.) Ball selon leur position géographique. Il s'agit là de la démonstration à partir de ces formations conifériennes d'arrière-dunes du phénomène classique de géosynvicariance.

Dans la mesure ou chaque communauté est suffisamment différencée par sa combinaison floristique, et possède une aire particulière, on peut parler d'association territoriale différente géosynvicariante. Il est commode de réunir ces associations géosynvicariantes en un groupe d'associations (suffixe eta) analogues. En l'occurence, il s'agit de Junipereta macrocarpae sur sable.

L'examen du tableau synthétique met en évidence quatre combinaisons d'espèces bien différenciées et possédant une aire distincte (cf. carte no 2 ). Ces quatre combinaisons ont à notre sens valeur d'associations.

La première combinaison, Sud-Ouest ibérique, correspond au Rhamno oleoidis-Juniperetum macrocarpae Riv.-Mart. 1964.

La deuxième combinaison, Nord africaine - Sicilienne, répond à l'Ephedro fragilis-Juniperetum macrocarpae Bartolo et al. 1982.

La troisième combinaison, adriatico-tyrrhénienne, est double et peut être référée à l'Asparago-Juniperetum macrocarpae de Bolòs 1964, à condition de préciser et de modifier ce nom en Asparago acutifolii-Juniperetum macrocarpae. Une légère variation floristique Nord-Sud de cette association permet d'y déceler deux races géographiques, l'une type plus septentrionale et l'autre plus méridionale à Prasium majus L. Cette dernière race correspond en synonymie au Pistacio-Juniperetum macrocarpae Caneva et al. 1981.

La quatrième combinaison, pontique, est celle du Marsdenio erectae-Juniperetum macrocarpae Géhu et Uslu 1989, dont il faudra à l'avenir relever plus de matériel.

Pour ce qui est de l'Egée, le matériel manque malheureusement et sur le terrain, nous n'avons jusqu'à présent rencontré que des individus isolés et relictuels de Juniperus oxycedrus L. ssp. macrocarpa (Sibth. et Sm.) Ball.

En Algérie, les limites de l'aire de l'Ephedro fragilis-Juniperetum macrocarpae Bartolo et al. 1982 restent à établir en raison des données contradictoires disponibles (Zaffran 1960, Quèzel et Santa 1962). 


\section{S C H E M A S Y N S Y S T EM A T I Q U E}

Querceceta ilicis Br.-B1. 1947

Pistacio-Rhamnetalia alaterni Riv. Mart. 1975

Juniperion lyciae Riv. Mart. 1975

Junipereta macrocarpae grp. ass. nov.

1. Rhamno (oleoidis)-Juniperetum macrocarpae Riv. Mart. 1964

2. Ephedro (fragilis)-Juniperetum macrocarpae Bartolo et al. 1982

3-4. Asparago-Juniperetum macrocarpae O. de Bolòs 1964

3. Race à Prasium majus nov. (syn. = Pistacio-Juniperetum macrocarpae Caneva et al. 1981)

4. Race type nov.

5. Marsdenio erectae-Juniperetum macrocarpae Gehu et Ulsu 1988

- Chacune de ces association possède probablement une sous association plus interne à Juniperus lycia.

\section{B I B L I O G R A P H I E}

ARRIGONI, P.V., NARDI, E. \& RAFFAELLI, M. -1985- La vegetazione del parco naturale della Maremma (Toscana). Univ. degli Studi di Firenze, Dipartimento di Biologia vegetale, 1-39. Firenze.

BARTOLO, G., BRULLO, S. \& MARCENO, C. -1982- La vegetazione costiera della Sicilia sud-orientale. Contributo alla interpretazione delle fasce di Vegetazione delle coste mediterranee. Collana del programma finalizzato "Promozione della qualita dell'ambiente", Consiglio Nat. del Rich. (AQ/1/226): 1-49 (Tab. H.T. 31). Roma.

BOLOS, O. (DE). -1962- El paisaje vegetal Barcelonés. Cátedra ciudad de Barcelona. Universidad de Barcelona. 1 vol. 192 pp. (Tab. 3). Barcelona.

BRAMBILLA, C., CANEVA, G., DE MARCO, G. \& MOSSA, L -1982- Analisi fitosociologia della seriazione psammofila costiera nella Sardegna meridionale. Annali di Botanica, 40:69-96 (Tab. 7). Roma.

CANEVA, G., MARCO, G. (DE) \& MOSSA, L. -1981- Analisi fitosociologica e cartografia della vegetazione (1/25 000) dell'isola di S. Antioco (Sardegna sud-occidentale). Collana del programma finalizzato "promozione della qualita dell'ambiente". Consiglio nazionale delle richerche, AQ/1/124: 1-59. (Tab. H.T. 10 ). Roma.

CORRIAS, B., DIANA-CORRIAS, L. \& VALSECHI, F. -1982- Carta della vegetazione della Nurra di Alghero (Sardegna nord-occidentale). Collana del programma finalizzato "promozione della qualita dell'ambiente". Consiglio nazionale delle richerche, AQ/1/229: 517. Roma.

GEHU, J.M., BIONDI, E., GEHU FRANK, J. \& MARCHIORI, S. -1984- Sur les tomillares à Thymus capitatus des dunes du Salento (Pouilles, Italie). Doc. Phytosoc. N.S. VIII:559565. (Tab. 4). Camerino.

GEHU, J.M., BIONDI, E., GEHU FRANCK, J. \& TAFFETANI, F. -1987- Donées sur la végétation maritime du littoral oriental de la Corse. V. Jornadas de fitosociología vegetación de Riberas de Agua Dulce. Ser. Inform., 22:363-391. (Tab. 7). Tenerife.

GEHU, J.M. \& GEHU FRANCK, J. -1986- Donnés synsystématiques et synchorologiques sur la végétation du littoral tunisien de Bizerte à Gabes. I. La végétation psammophile. Doc Phytosoc., N.S. X (II): 127-155. (Tab. 15). Camerino.

MAUGERI, G. \& LEONARDI, S. -1974- Esempio di macchia a Ginebro e lentisco nella Sicilia meridionale. Publicazioni dell'Istituto di Botanica dell'Universita di Catania. pp. 51- 
59. Forli.

QUEZEL, P. \& SANTA, S. -1962- Nouvelle flore de l'Algérie. Tome 1, p. 36. C.N.R.S., Paris.

RIVAS MARTINEZ, S. -1964- Esquema de la vegetación potencial y su correspondencia con los suelos en la España peninsular. Anales del Instituto Botánico A.J. Cavanilles. XXII:341-405. Madrid.

RIVAS MARTINEZ, S. -1980- Estudio botánico de los ecosistemas de Doñana (Huelva, España). Lazaroa, 2:3-4. Madrid.

(Aceptado para su publicación el 2 de marzo de 1990)

Dirección de los autores: J.M. GEHU: Université de Paris V - 4, Avenue de l'Observatorie - F75270 Paris et Centre Régional de Phytosociologie, F-59270 Bailleul - France. M. COSTA: Universidad de Valencia. Avda Blasco ibáñez, 13 - E-46010 Valencia. España. E. BIONDI: Universita dell'Aquila - Piazza dell'annunziata, 1 - I67100 l'Aquila. Italie. 
TABLEAU SYNSYSTEMATIQUE DE SYNTHESE

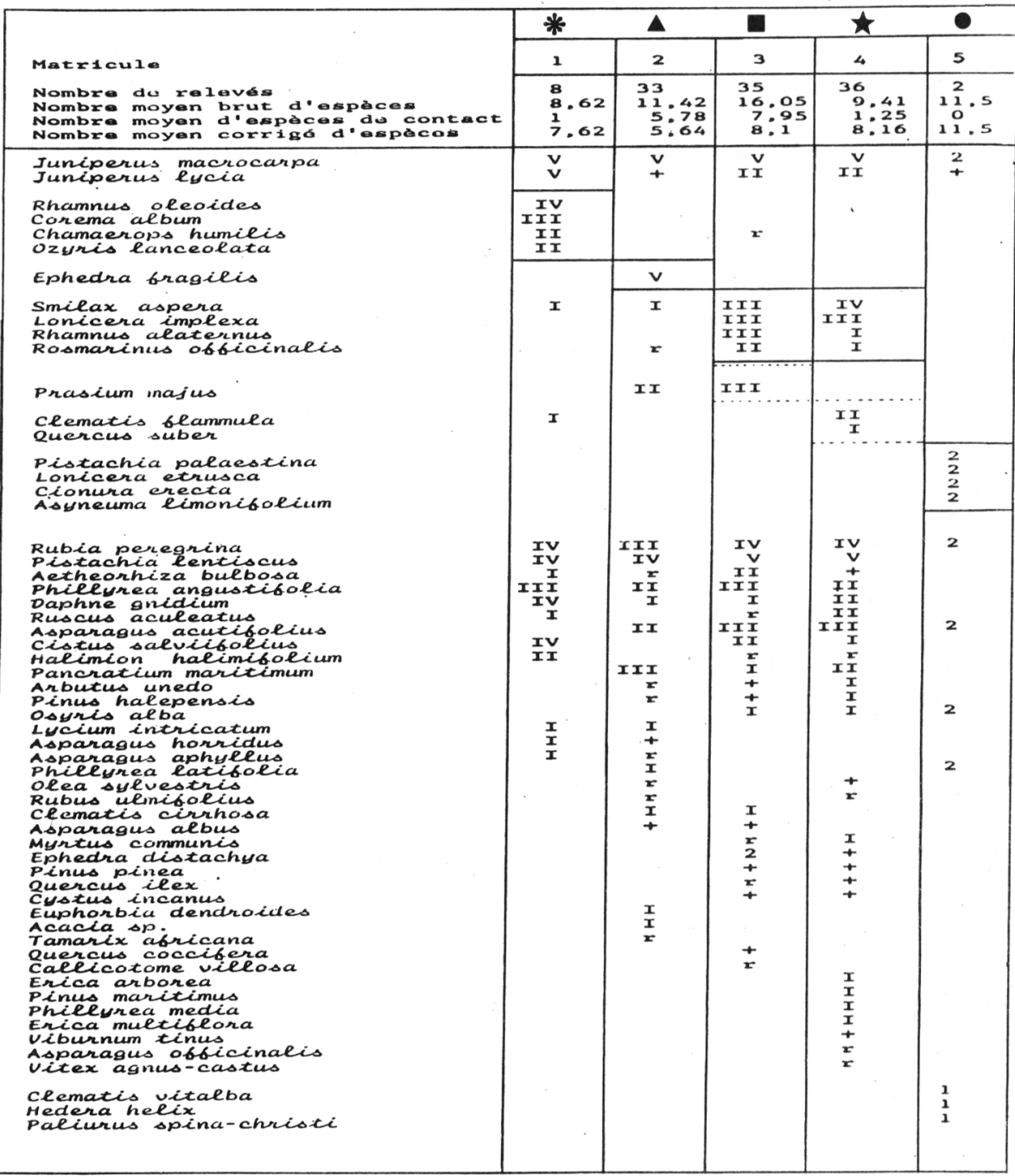

LEGENDE. 1, Rhamno oleoidis-Juniperetum incacrocarpae. 2, Ephedro fragilisJuniperetum macrocarpae. 3-4, Asparago-Juniperetum macrocarpae. 3, Race à Prasium majus. 4, Race type. 5, Marsdenio (cyonuro)-Juniperetum macrocarpae

S O U R C E S . 1, Rivas Martínez 1964 (2 Rel.); Rivas Martínez 1980 (6 Rel). 2, Maugeri et Leonardi 1974 (12 Rel.), M. Bartolo et al. 1982 (15 Rel.), Gehu et Gehu Franck (6 Rel.). 3, Brambilla et al. 1982 (25 Re.), Caneva et al. 1981 (5 Rel.), Gehu et al 1984 (5 Rel.). 4, De Bolòs 1962 (4 Rel.), Gehu et al (21 Rel. ined.), Gehu et al. 1987 (10 Rel.), Corrias et al. (1 Rel.). 5. Gehy et Uslu 1988 (2 Rel.). 


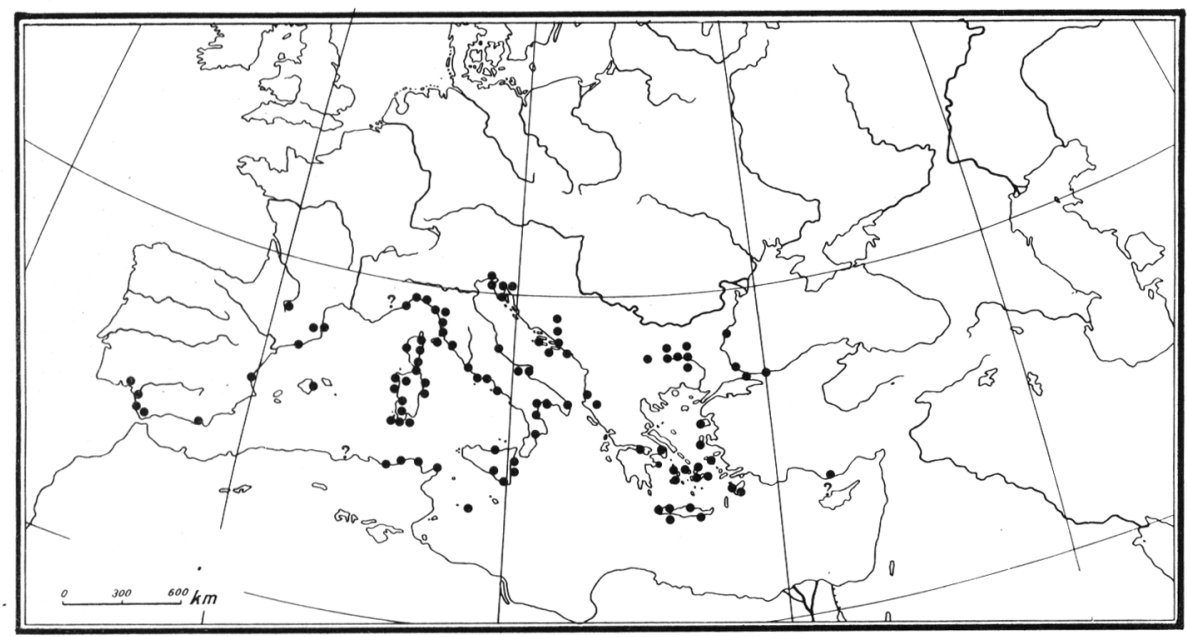

CARTE № 1: Juniperus oxycedrus subsp. macrocarpa (selon Atlas Florae Europaeae modifié)

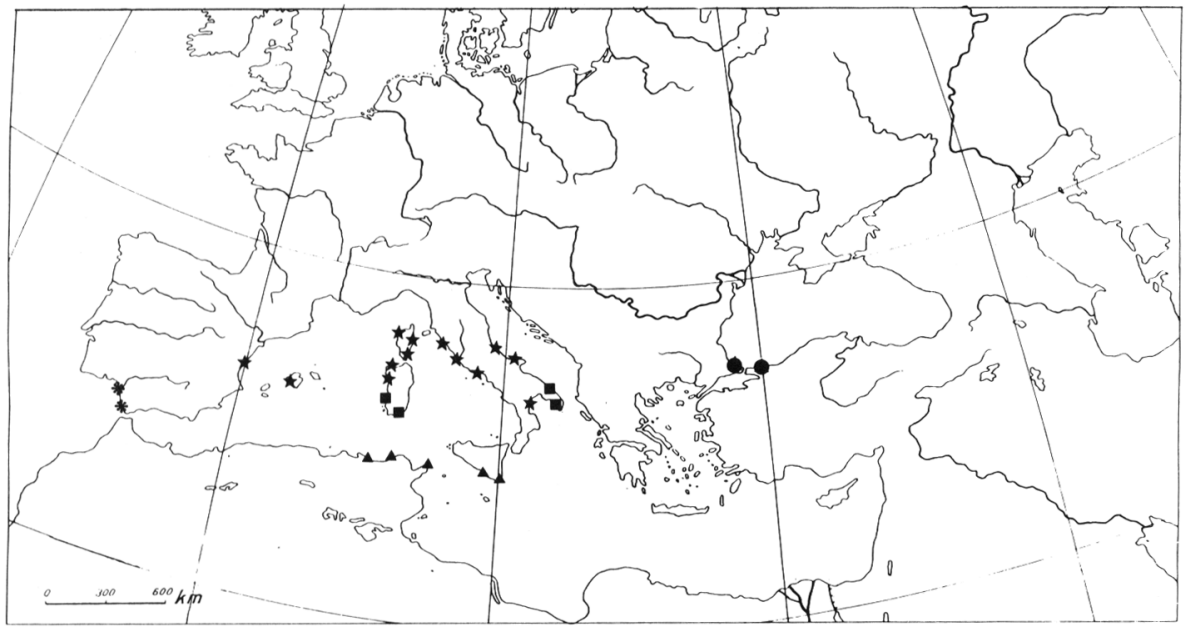

CARTE №2: Géosynvicariance des. Junipereta macrocarpae. 